\title{
Quasi-Dirac neutrinos in the linear seesaw model
}

\author{
Kevin Monsálvez-Pozo, ${ }^{a, *}$ Carolina Arbeláez, ${ }^{b}$ Claudio Dib $^{b}$ and Iván Schmidt ${ }^{b}$ \\ ${ }^{a}$ Instituto de Física Corpuscular, CSIC - Universitat de València, \\ Apt. Correus 22085, E-46071 València, Spain \\ ${ }^{b}$ Universidad Técnica Federico Santa María and Centro Científico Tecnológico de Valparaíso CCTVal, \\ Avenida España 1680, Valparaíso, Chile \\ E-mail: kevin.monsalvez@ific.uv.es, carolina.arbelaez@usm.cl, \\ claudio.dib@usm.cl, ivan.schmidt@usm.cl
}

\begin{abstract}
We implement a minimal linear seesaw model (LSM) for addressing the Quasi-Dirac (QD) behaviour of heavy neutrinos, focusing on the mass regime of $M_{N} \lesssim M_{W}$. Here we show that for relatively low neutrino masses, covering the few $\mathrm{GeV}$ range, the same-sign to opposite-sign dilepton ratio, $R_{\ell \ell}$, can be anywhere between 0 and 1, thus signaling a Quasi-Dirac regime. Particular values of $R_{\ell \ell}$ are controlled by the width of the QD neutrino and its mass splitting, the latter being equal to the light-neutrino mass $m_{v}$ in the LSM scenario. The current upper bound on $m_{v_{1}}$ together with the projected sensitivities of current and future $\left|U_{N \ell}\right|^{2}$ experimental measurements, set stringent constraints on our low-scale QD mass regime. Some experimental prospects of testing the model by LHC displaced vertex searches are also discussed.
\end{abstract}

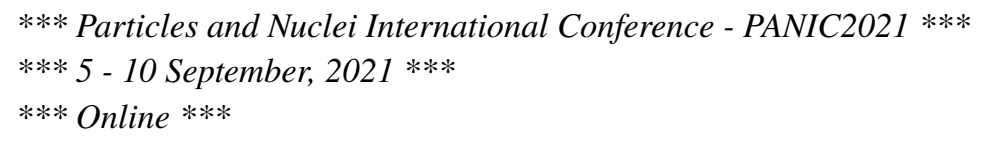

${ }^{*}$ Speaker 


\section{Introduction}

Neutrino oscillation experiments have found overwhelming evidence for the existence of non vanishing -albeit small- neutrino masses, thus showing the existence of Physics Beyond the Standard Model. The most widely accepted mechanism to generate small neutrino masses is the well-known seesaw mechanism, which involves extra heavy neutral fermions, denoted here as $N_{i}$ ( $i=1,2, \ldots, n$, depending on the model). In most of these scenarios, the heavy neutrinos are Majorana fermions. However, there are seesaw scenarios where pairs of these Majorana neutrinos approach smoothly their mass degeneracy limit, $\Delta M_{N} \rightarrow 0$. These almost degenerate Majorana neutrinos are usually called Quasi-Dirac (QD) neutrinos. In the recent work in Ref. [1], we studied the framework of the minimal linear seesaw model, which naturally yields pairs of Quasi-Dirac right-handed neutrinos $N$ and $N^{\prime}$ in a regime of masses below $M_{W}$.

\section{Model setup}

Besides the SM content, the minimal version of the LSM contains two different types of neutral $S U(2)$ singlet fermions $(N, S)$ per generation. Considering the basis $\left(v_{L}^{c}, N, S\right)$, the texture of the neutrino mass $(9 \times 9)$ matrix, given in a $3 \times 3$ block notation, reads:

$$
M_{v}=\left(\begin{array}{ccc}
0 & m_{D} & M_{\epsilon} \\
m_{D}^{T} & 0 & M_{R} \\
M_{\epsilon}^{T} & M_{R}^{T} & 0
\end{array}\right),
$$

where $m_{D}=v_{S M} Y_{D} / \sqrt{2}$ and $M_{\epsilon}=v_{S M} Y_{\epsilon} / \sqrt{2}$, with $Y_{D}$ and $Y_{\epsilon} 3 \times 3$ Yukawa matrices and $M_{R}$ being a $3 \times 3$ diagonal matrix. Bringing this $3 \times 3$ matrix into a block-diagonal form - through a diagonalization-like procedure considering $M_{\epsilon} \ll m_{D}<M_{R}$ - the non-diagonal mass matrix of the light neutrinos is given by

$$
m_{\nu}=m_{D} M_{R}^{-1} M_{\epsilon}^{T}+M_{\epsilon} M_{R}^{T^{-1}} m_{D}^{T}=\frac{v^{2}}{2}\left(Y_{D} M_{R}^{-1} Y_{\epsilon}^{T}+Y_{\epsilon} M_{R}^{T^{-1}} Y_{D}^{T}\right)
$$

The smallness of the lepton-number violating term $M_{\epsilon}$, together with the linearity on $m_{D}$, generates small $m_{v}$ masses for the light neutrinos, without requiring $M_{R}$ to be extremely large.

An important feature of the LSM is that the mass splitting between the two heavy neutrinos within each generation is very small:

$$
\Delta M_{i} \sim m_{v_{i}},
$$

This is crucial in order to study the Quasi-Dirac behaviour of pairs of heavy neutrinos in the linear seesaw model, as it is described in the next section.

\section{Quasi-Dirac neutrinos}

The usual Dirac-Majorana dichotomy regarding the nature of neutrinos is somehow misleading, since the Dirac case can be considered as a limiting case of a more general Majorana scenario with twice the neutrino content and degenerated masses, where all LNV sources of the model have vanished. 
An observable that is commonly used at the LHC to look for Majorana neutrinos is the samesign (SS) to opposite-sign (OS) dilepton ratio in $\ell \ell j j$ events with no missing $p_{T}$, which we call $R_{\ell \ell}$. The production of these events with a pair of leptons of the same sign, are expected to occur only through lepton number violating processes mediated by Majorana neutrinos, while opposite-sign pair of leptons are produced via lepton number conserving processes mediated by both Dirac and Majorana neutrinos.

The ratio of SS over OS events can be expressed as [2]:

$$
R_{\ell \ell}=\frac{\Delta M^{2}}{2 \Gamma^{2}+\Delta M^{2}} .
$$

Note that $R_{\ell \ell} \rightarrow 1$ if $\Gamma \ll \Delta M$, while $R_{\ell \ell} \rightarrow 0$ if $\Delta M \ll \Gamma$. These two scenarios correspond to the limiting Majorana and Dirac cases, respectively. In models with Quasi-Dirac neutrinos, the ratio $R_{\ell \ell}$ can take any value between 0 and 1; as we approach $R_{\ell \ell} \rightarrow 0$, the LNV effects are more and more suppressed. Using the estimate for the mass splitting from Eq. (3), it follows that the window of $R_{\ell \ell}$ values compatible with QD neutrinos is determined, in the linear seesaw model, by the light-neutrino masses and the heavy-neutrino decay width.

\section{Parametrization}

We intended to cover in the most general way the parameter space of the linear seesaw, focusing on the regions where current and near-future experiments aim to explore. For this purpose, we took the master parametrization described in Ref. [3] for the concrete case of the linear seesaw, which allows to fit any Majorana neutrino mass model and automatically reproduce current experimental data.

\section{Results}

\subsection{Dilepton ratio in the LSM}

The Quasi-Dirac regime $0<R_{\ell \ell}<1$ occurs when $\Delta M \sim \Gamma$. Since $\Delta M \sim m_{v}$ and $\Gamma\left(M_{N}\right)$ grows quite fast with $M_{N}$, for smaller values of $m_{v_{1}}$, smaller values of $M_{N}$ are needed. This is illustrated in Fig. 1, which shows the regions in the $m_{v_{1}}-M_{N_{1}}$ plane that belong to the QD regime. As expected, for each specific $m_{v_{1}}$, there is a relatively narrow window of $M_{N_{1}}$ values such that $0<R_{\ell \ell}<1$. For example, if $m_{v_{1}}=10^{-5} \mathrm{eV}$, then values of $10 \mathrm{GeV} \lesssim M_{N_{1}} \lesssim 20 \mathrm{GeV}$ are needed in order to obtain a $R_{\ell \ell}$ value within the QD regime. Unlike the inverse seesaw model [2], where values of $R_{\ell \ell}<1$ are still obtained for larger values of $M_{N_{1}}$, here the current upper bound for the light neutrino mass $m_{v_{1}} \lesssim 0.1 \mathrm{eV}$ and a $f^{\prime}=100$ sets $R_{\ell \ell}=0$ for values of $M_{N_{1}} \gtrsim 100 \mathrm{GeV}$.

\subsection{Heavy to light neutrino mixing $U_{N} \ell$}

We analyzed the $\left|U_{N_{1} \ell}\right|^{2}-M_{N_{1}}$ region and identified the zones in the parameter space where some current experiments could have sensitivity; the prospects of future experiments were also considered. The numerical analysis was based on the systematic diagonalization of the $9 \times 9$ mass matrix of the neutral states $M_{v}$ (see Eq. (1)) 


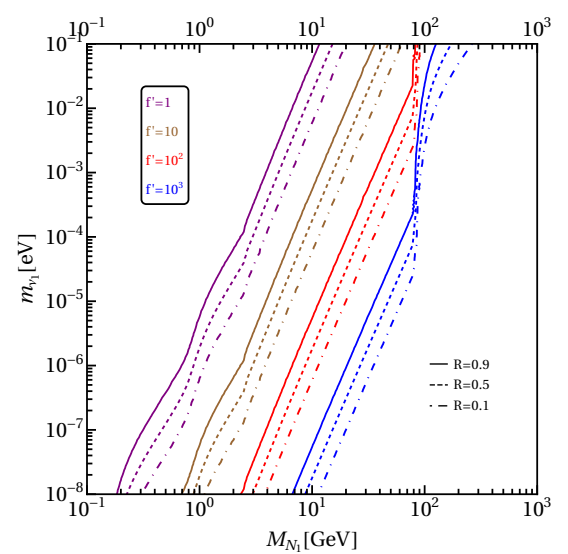

Figure 1: $m_{v_{1}}-M_{N_{1}}$ lines corresponding to a specific value of $R_{\ell \ell}$ and $f^{\prime}$.

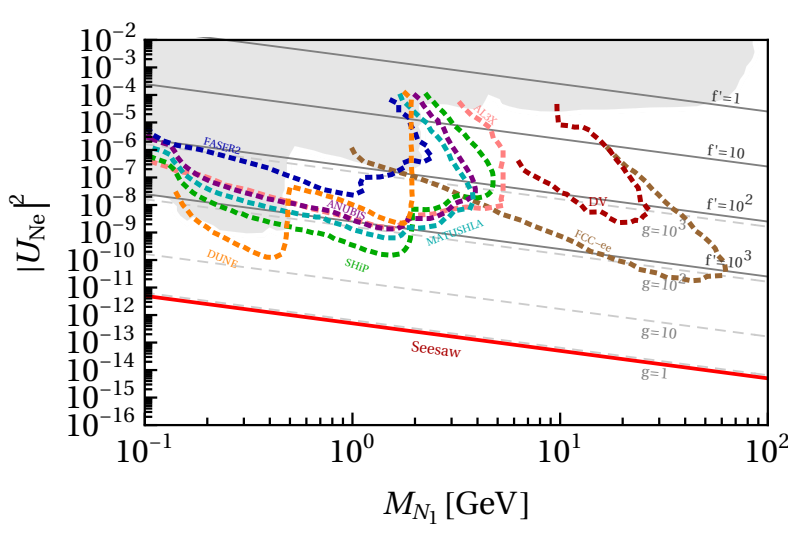

Figure 2: Active-sterile neutrino mixing $\left|U_{N_{1} e}\right|^{2}$ versus the neutrino mass $M_{N_{1}}$, for different values of the parameters $f^{\prime}$ and $g$.

$$
M_{v}=U \hat{M}_{v} U^{T},
$$

with $U$ containing the PMNS mixing matrix and the heavy-light neutrino mixing elements $U_{N_{1} \ell}$ as well.

We present the results in Fig. 2. Some of our findings are summarized as follows:

- Scenario a: all neutrino masses $m_{v_{1}}$ enter all $Y_{\epsilon}$ entries, while $Y_{D}$ is independent of the light neutrino masses. Then the mixing $U_{N_{1} e}$ does not depend on $m_{v_{1}}$.

- Scenario $b$ : light neutrino masses enter separately all $Y_{\epsilon}$ and $Y_{D}$ entries, an explicit dependence on $m_{v_{1}}$ is then expected. This results in a less constrained scenario.

- Scenario a and $b$ : if there is some appreciable hierarchy between the Yukawas $Y_{\epsilon}$ and $Y_{D}$, the predicted mixing falls into the range testable by present and near-future experiments.

The bounds on the mixing themselves can place already stringent constraints into the QD regime, which can be found by studying the interplay between Figures 1 and 2 .

\section{Acknowledgements}

This work was supported in part by FONDECYT (Chile) grants No. 11180722, 1180232 and 1170171, and by ANID (Chile) PIA/APOYO AFB 180002, as well as by Grants No. FPA201784445-P and SEV-2014-0398 (AEI/ERDF, EU) and by PROMETEO/2017/053 (GV).

\section{References}

[1] C. Arbeláez, C. Dib, K. Monsálvez-Pozo and I. Schmidt, Quasi-Dirac neutrinos in the linear seesaw model, JHEP (2021) 154 [2104.08023].

[2] G. Anamiati, M. Hirsch and E. Nardi, Quasi-Dirac neutrinos at the LHC, JHEP 10 (2016) 010 [1607.05641].

[3] I. Cordero-Carrión, M. Hirsch and A. Vicente, Master Majorana neutrino mass parametrization, Phys. Rev. D 99 (2019) 075019 [1812 .03896]. 